\title{
Período Seco Anterior e Período de Serviço sobre a Produção de Leite e Gordura na Raça Holandesa no Estado de Minas Gerais ${ }^{1}$
}

\author{
Antonio Ilson Gomes de Oliveira², Idalmo Garcia Pereira ${ }^{3}$, Tarcísio de Moraes Gonçalves ${ }^{4}$, \\ José Vânio Araújo ${ }^{5}$
}

RESUMO - Para estudar efeitos dos períodos seco anterior (PSA) e de serviço (PSER) sobre produção de leite (L305) e gordura (G305) em até 305 dias de lactação e produção de leite (LDIA) e gordura (GDIA) por dia de intervalo de parto em vacas holandesas, 5454 lactações foram analisadas, provenientes dos arquivos de Controle Leiteiro da ACGHMG. Efeitos de núcleo (5), rebanho-ano:núcleo, grupo genético (1- 31/32 Holandês, 2- PC com geração controlada, GC's, e 3- PO), estação-idade ao parto em classe, período seco anterior (28 classes), período de serviço (21 classes) e duração da lactação (efeito linear) foram considerados no modelo. As médias, os erros-padrão e coeficientes de variação para L305, G305, LDIA e GDIA foram, respectivamente, $5.721,60(13,87 \mathrm{~kg})-\mathrm{CV}=17,90 \%, 191,04(0,46 \mathrm{~kg})-\mathrm{CV}=18,20 \%, 15,04(0,04 \mathrm{~kg})-\mathrm{CV}=18,02 \% \mathrm{e}$ $0,5041(0,0011 \mathrm{~kg})-\mathrm{CV}=17,66 \%$. Maior PSER foi associado à menor L305, G305, LDIA e GDIA, definidos pelas equações: L305 $=5.982,93-4,3042$ PSER $\left(\mathrm{R}^{2}=85,15 \%\right)$ e LDIA $=19,4569-0,04289$ PSER $\left(\mathrm{R}^{2}=98,87 \%\right)$. As relações entre estas características com as médias das classes de PSA foram descritas por equações cúbicas: L305 = 4.910,70 + 14,0799 PSA - 0,0877 $\mathrm{PSA}^{2}+0,00016 \mathrm{PSA}^{3}\left(\mathrm{R}^{2}=53,99 \%\right)$ e LDIA $=12,62+0,0396 \mathrm{PSA}-0,00025 \mathrm{PSA}^{2}+0,00000045 \mathrm{PSA}^{3}\left(\mathrm{R}^{2}=56,58 \%\right)$. Os demais fatores também influenciaram todas as características avaliadas. O ajustamento, principalmente de LDIA e GDIA para período de serviço, deverá ser importante nas avaliações genéticas.

Palavras-chave: produção de leite e gordura em até 305 dias, produção por dia de intervalo de parto

\section{Previous Days Dry and Days Open on Milk and Fat Yield in the Holstein Cows of Minas Gerais State - Brazil}

\begin{abstract}
To evaluate the effect of previous days dry (PDD) and days open (DO) on milk (M305) and fat (F305) yield in 305 days and milk (MDAY) and fat (FDAY) yield per day of calving interval in Holstein cows, a data set contained 5,454 lactations from the Minas Gerais Holstein Cattle Breeders Association were analyzed. The model included effects of: nucleus (5), heard-year: nucleus, genetic group (1-31/32 Holstein, 2- upgraded Holstein with known genealogy and 3- pure of origin Holstein), season-calving age in classes, previous days dry (28 classes), days open (21 classes) and lactation length (linear

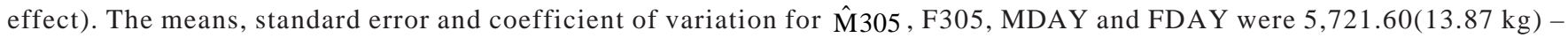
CV, 17.90\%, 191.04(0.46 kg) - CV, 18.20\%, 15.04(0.04 kg) - CV, 18.02\% and .5041 (.0011kg) - CV, 17.66\%, respectively. Higher DO were related to lower M305 and F 305 and MDAY and FDAY, described by equations M305 = 5,982.93 - 4.3042 $\mathrm{DO}\left(\mathrm{R}^{2}=85.15 \%\right)$ and $\hat{\mathrm{MDAY}}=19.4569-0.04289 \mathrm{DO}\left(\mathrm{R}^{2}=98.87 \%\right)$. The relations among this traits with the means of PDD classes were described by cubic equations: $\hat{\mathrm{M}} 305=4,910.70+14.0799 \mathrm{PDD}-0.0877 \mathrm{PDD}^{2}+0.00016 \mathrm{PDD}^{3}\left(\mathrm{R}^{2}=53.99 \%\right)$ and $\mathrm{MDAY}=12.62+0.0396 \mathrm{PDD}-0.00025 \mathrm{PDD}^{2}+0.00000045 \mathrm{PDD}^{3}\left(\mathrm{R}^{2}=56.58 \%\right)$. Significant effect of all the other factors in all the evaluated traits was observed. The adjustment, mainly for MDAY and FDAY for days open, may be important in the genetic evaluations.
\end{abstract}

Key Words: 305-days milk and fat yield, yield by day calving interval

\section{Introdução}

Estudos realizados no sentido de verificar a influência do período seco anterior sobre a produção de leite e gordura, em até 305 dias de lactação, têm demonstrado o efeito significativo desta característica. Por outro lado, o período de serviço é a medida que oferece a mais significativa análise da condição reprodutiva de uma vaca, ou mesmo de um rebanho (OLIVEIRA FILHO et al., 1985). Dessa forma, este parâmetro tem influência direta na vida produtiva da vaca, uma vez que aumento em sua duração eleva o intervalo de partos, com conseqüente redução do número de crias por rebanho, acarretando prejuízo à

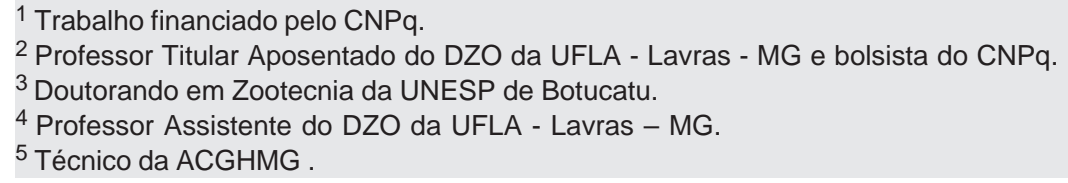


exploração leiteira e comprometendo a rentabilidade (LOUCA e LEGATES, 1968).

Entre os autores que verificaram efeito significativo do período seco anterior sobre a produção de leite e gordura em até 305 dias, destacam-se os trabalhos de SCHAEFFER e HENDERSON (1972) e SMITH e LEGATES (1962). No Brasil, TEIXEIRA e VALENTE (1994) relataram que, do ponto de vista econômico, a produção de leite por unidade de tempo é considerada variável importante, por ser influenciada pelo período seco anterior. No trabalho desses autores, a produção foi expressa como produção anualizada, a qual é uma alternativa de se expressar a produção de leite por dia de intervalo de parto.

Alguns autores têm demonstrado efeito significativo do período de serviço sobre a produção de leite e gordura em até 305 dias de lactação (SCHAEFFER e HENDERSON, 1972; TEIXEIRA et al., 1995; e THOMPSON et al., 1982). Entre os poucos trabalhos encontrados com relação ao efeito do período de serviço sobre a produção por dia de intervalo de parto, verificou-se que esta produção expressa na forma anualizada é influenciada pelo período de serviço (TEIXEIRA e VALENTE, 1994).

As estimativas de herdabilidade para período seco anterior $(0,15$ a 0,34 , segundo SCHAEFFER e HENDERSON, 1972) demonstram que esta caraterística sofre certa influência ambiental. Nesse caso, esses autores não recomendam fatores de ajustamento para período seco anterior, visto que isto poderia resultar em vícios na estimativa do valor genético. Mais recentemente, entretanto, VALENTE et al. (1995) sugeriram que o ajustamento para o período seco seria recomendável, quando utilizado em avaliações genéticas. O período de serviço apresenta valores de herdabilidade ainda menores, inferiores a 0,10 (THOMPSON et al., 1982). Outro trabalho (TEIXEIRA e VALENTE, 1994) tem concluído que esta característica, por ser amplamente influenciada pelo ambiente, necessita de ajustamento, não introduzindo vícios na estimativa do valor genético, o que está de acordo também com KHATTAB e ASHMAWY (1988), SMITH e LEGATES (1962) e THOMPSON et al. (1982).

O objetivo deste estudo foi verificar a influência do período seco anterior e do período de serviço sobre a produção de leite e gordura em até 305 dias e a produção por dia de intervalo de parto em vacas da raça holandesa no Estado de Minas Gerais.

\section{Material e Métodos}

As observações utilizadas neste estudo são provenientes dos arquivos de Controle Leiteiro da ACGHMG (Associação de Criadores de Gado Holandês de Minas Gerais). Um total de 49.276 lactações foi previamente avaliado, mantendo-se para análise registros de rebanhos que apresentassem pelo menos cinco lactações e estivessem presentes em pelo menos dois anos; registros com período seco entre 0 e 300 dias; registros com período de serviço entre 20 e 229 dias; registros com intervalo de partos entre 280 e 720 dias; registros com idade ao parto entre 20 e 200 meses; registros com duração de lactação entre 150 e 450 dias; e registros de lactações com produção superior a $1000 \mathrm{~kg}$ de leite. Após esta etapa, foram aproveitados 5454 registros para o estudo dos fatores incluídos no modelo.

Foram estudados os dados dos núcleos de criadores da ACGHMG, localizados em diversas regiões do Estado, que controlam as informações de rebanhos daquela região. Os dados foram preparados e analisados utilizando-se o programa computacional "SAS", considerando-se no modelo, como variáveis independentes, os efeitos de núcleo (5), rebanho-ano:núcleo, grupo genético (1- 31/32 Holandês, 2- PC com geração controlada, GC's, e 3- PO), estação-idade ao parto em classes (duas estações - águas de outubro a março e seca de abril a setembro - e 19 classes de idade), período seco anterior (PSA), período de serviço (PSER) e duração da lactação (efeito linear) e, como variáveis dependentes, a produção de leite e gordura em até 305 dias e a produção de leite e gordura por dia de intervalo de parto. As classes de período seco anterior totalizaram 28 e as de período de serviço, 21 (Tabela 1). Os fatores de ajuste foram estimados com base na média (114,6 dias) dos PSER, dividindo-se todas as produções estimadas pela equação de regressão pela produção estimada aos 114 dias de PSER.

\section{Resultados e Discussão}

Médias, erros-padrão e coeficientes de variação para a produção de leite (L305) e gordura (G305) em até 305 dias foram $5.721,60 \pm 13,87 \mathrm{~kg}$ e $17,90 \%$ e $191,04 \pm 0,46 \mathrm{~kg}$ e $18,20 \%$, respectivamente. As classes de período de serviço (PSER) e de período seco anterior (PSA) influenciaram (Tabela 2) tanto L305 como G305, sendo semelhantes aos resultados obtidos por SCHAEFFER e HENDERSON (1972), 
1018 Rev. bras. zootec.

Tabela 1 - Classe de período de serviço e de período seco anterior, com respectivos intervalos e número de observações Table 1 - Days open and previous days dry classes with respective intervals and records number

\begin{tabular}{|c|c|c|c|c|}
\hline $\begin{array}{l}\text { Classe } \\
\text { Class }\end{array}$ & $\begin{array}{c}\text { Intervalo de período } \\
\text { de serviço (dias) } \\
\text { Days open inteval (days) }\end{array}$ & $\begin{array}{l}\text { Número de observações } \\
\text { Records number }\end{array}$ & $\begin{array}{c}\text { Intervalo de período } \\
\text { seco anterior (dias) } \\
\text { Previous days dry intevals (days) }\end{array}$ & $\begin{array}{c}\text { Número de observações } \\
\text { Records number }\end{array}$ \\
\hline 1 & $20-29$ & 35 & $0-9$ & 43 \\
\hline 2 & $30-39$ & 65 & $10-19$ & 56 \\
\hline 3 & $40-49$ & 165 & $20-29$ & 101 \\
\hline 4 & $50-59$ & 327 & $30-39$ & 225 \\
\hline 5 & $60-69$ & 428 & $40-49$ & 343 \\
\hline 6 & $70-79$ & 498 & $50-59$ & 512 \\
\hline 7 & $80-89$ & 493 & $60-69$ & 632 \\
\hline 8 & $90-99$ & 469 & $70-79$ & 576 \\
\hline 9 & $100-109$ & 388 & $80-89$ & 504 \\
\hline 10 & $110-119$ & 372 & $90-99$ & 439 \\
\hline 11 & $120-129$ & 349 & $100-109$ & 314 \\
\hline 12 & $130-139$ & 290 & $110-119$ & 271 \\
\hline 13 & $140-149$ & 254 & $120-129$ & 247 \\
\hline 14 & $150-159$ & 231 & $130-139$ & 180 \\
\hline 15 & $160-169$ & 194 & $140-149$ & 143 \\
\hline 16 & $170-179$ & 185 & $150-159$ & 103 \\
\hline 17 & $180-189$ & 162 & $160-169$ & 95 \\
\hline 18 & 190-199 & 170 & $170-179$ & 88 \\
\hline 19 & $200-209$ & 141 & $180-189$ & 76 \\
\hline 20 & $210-219$ & 130 & $190-199$ & 71 \\
\hline 21 & $220-229$ & 108 & $200-209$ & 69 \\
\hline 22 & - & - & $210-219$ & 65 \\
\hline 23 & - & - & $220-229$ & 57 \\
\hline 24 & - & - & $230-239$ & 48 \\
\hline 25 & - & - & $241-251$ & 46 \\
\hline 26 & - & - & $252-265$ & 56 \\
\hline 27 & - & - & $266-281$ & 53 \\
\hline 28 & - & - & $282-300$ & 41 \\
\hline
\end{tabular}

SMITH e LEGATES (1962) e THOMPSON et al. (1982). O período de serviço contribuiu com cerca de 1,35 e 1,60\% da variação total em L305 e G305, respectivamente - valor inferior aos encontrados por SMITH e LEGATES (1962).

Rebanho-ano:núcleo foi o fator que mais contribuiu na variação total da produção de leite e gordura em até 305 dias (30,6 e 29,12\%), o que possivelmente é atribuído ao fato de o nível de manejo estar caracterizado por rebanho e ano dentro de determinado núcleo (regiões do Estado de Minas Gerais geograficamente distintas que proporcionam condições bastante diferenciadas para a expressão destas características de produção). O segundo fator que mais contribuiu (15,04 e 16,05\%) foi a duração da lactação (efeito linear), pois, quando se considera a produção em até 305 dias, existe grande número de lactações inferiores a este período que não foram projetadas para 305 dias e, dessa forma, o aumento na duração da lactação leva a aumento na produção.

Médias, erros-padrão e coeficientes de variação para LDIA e GDIA foram 15,04 $\pm 0,04 \mathrm{~kg}$ e $18,02 \%$ e $0,5041 \pm 0,0011 \mathrm{~kg}$ e $17,66 \%$, respectivamente. A LDIA e GDIA também foram influenciadas pelo efeito do PSER (12 a $13 \%$ da variação total) e do PSA (Tabela 3).

As demais fontes de variação também influenciaram LDIA e GDIA (Tabela 3). Nesta análise, os fatores que mais contribuíram para variação na LDIA e GDIA foram os mesmos citados para L305 e G305, porém, estes fatores, especialmente rebanhoano:núcleo, participaram em porcentagens diferentes (27 e 20\%, respectivamente). Isto possivelmente se explica pela contribuição maior do PSER na variação destas produções, ou seja, PSER parece exercer maior influência (medida em termos de $\mathrm{R}^{2}$ ) nas produções por dia de intervalo de parto que nas produções em até 305 dias, sugerindo que estas produções devem ser ajustadas para o efeito de PSER.

As relações entre L305 e PSER foram descritas por equações lineares decrescentes (Figura 1) e foram similares às encontradas entre G305 e PSER, o que se assemelha com as relações encontradas por KHATTAB e ASHMAWY (1988). No entanto, 
OLIVEIRA et al.

Tabela 2 - Análise de variância das produções de leite (L305) e gordura (G305) em 305 dias

Table 2 - Analysis of variance of milk (M305) and fat (F305) yields in 305 days

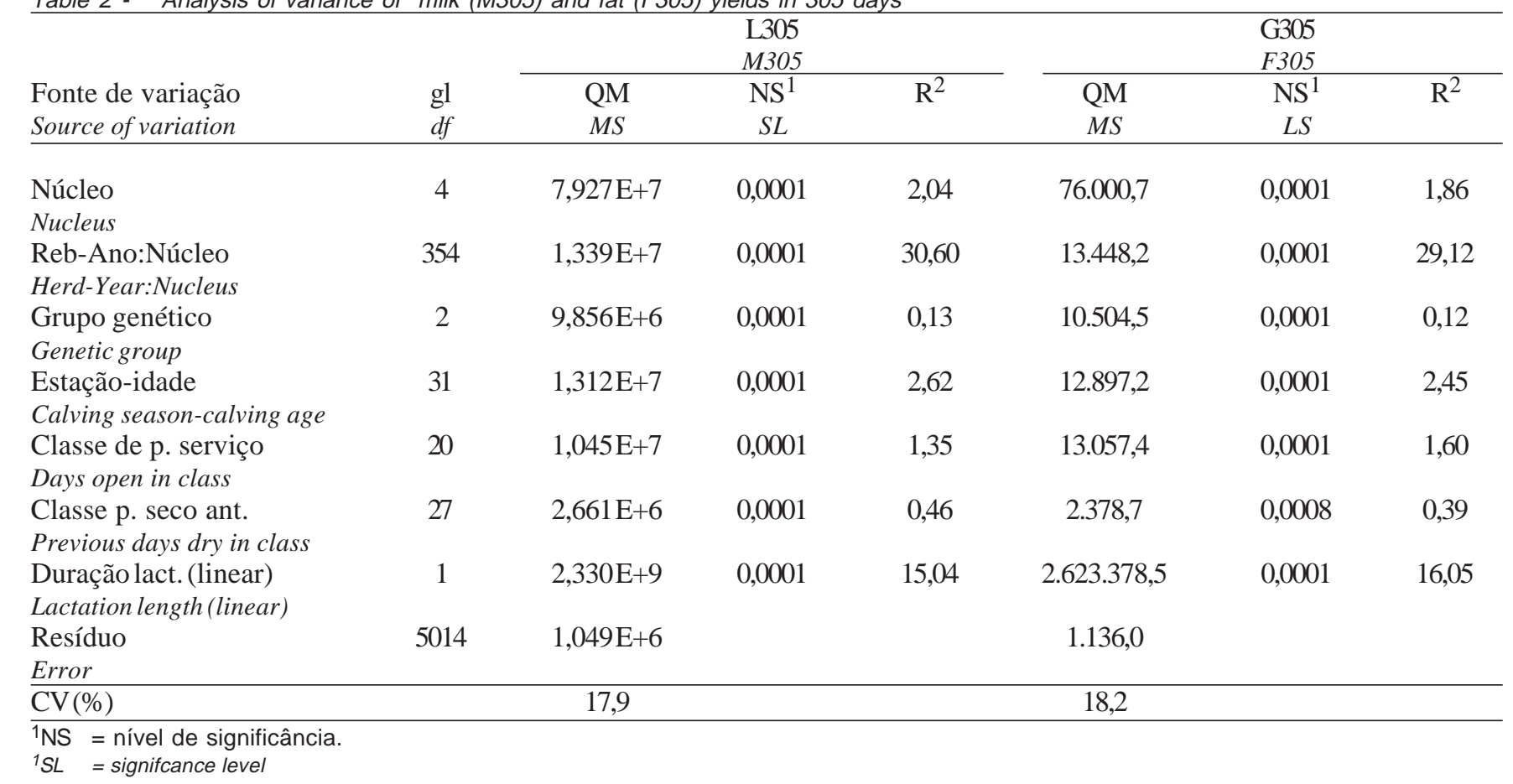

Tabela 3 - Análise de variância das produções de leite (LDIA) e gordura (GDIA) por dia de intervalo de parto Table 3 - Analysis of variance of milk (MDAY) and fat yields (FDAY) by day of calving interval

\begin{tabular}{|c|c|c|c|c|c|c|c|}
\hline \multirow[b]{2}{*}{ Fonte de variação } & \multirow[b]{2}{*}{$\mathrm{gl}$} & \multicolumn{3}{|c|}{$\begin{array}{l}\text { LDIA } \\
M D A Y\end{array}$} & \multicolumn{3}{|c|}{$\begin{array}{l}\text { GDIA } \\
F D A Y\end{array}$} \\
\hline & & QM & $\mathrm{NS}^{1}$ & $\mathrm{R}^{2}$ & QM & $\mathrm{NS}^{1}$ & $\mathrm{R}^{2}$ \\
\hline Source of variation & $d f$ & $M S$ & $S L$ & & $M S$ & & \\
\hline Núcleo & 4 & 539,613 & 0,0001 & 1,84 & 0,5295 & 0,0001 & 1,68 \\
\hline Nucleus & & & & & & & \\
\hline $\begin{array}{l}\text { Reb-Ano:Núcleo } \\
\text { Herd-Year:Nucleus }\end{array}$ & 354 & 92,512 & 0,0001 & 27,87 & 0,0919 & 0,0001 & 25,87 \\
\hline $\begin{array}{l}\text { Grupo genético } \\
\text { Genetic group }\end{array}$ & 2 & 70,361 & 0,0001 & 0,12 & 0,0770 & 0,0001 & 0,12 \\
\hline $\begin{array}{l}\text { Estação-idade } \\
\text { Calving season-calving age }\end{array}$ & 31 & 86,111 & 0,0001 & 2,27 & 0,0845 & 0,0001 & 2,08 \\
\hline $\begin{array}{l}\text { Classe de p. serviço } \\
\text { Days open in class }\end{array}$ & 20 & 732,971 & 0,0001 & 12,48 & 0,8348 & 0,0001 & 13,27 \\
\hline $\begin{array}{l}\text { Classe p. seco ant. } \\
\text { Previous days dry in class }\end{array}$ & 27 & 19,546 & 0,0001 & 0,45 & 0,0176 & 0,0003 & 0,38 \\
\hline $\begin{array}{l}\text { Duração lact. (linear) } \\
\text { Lactation length(linear) }\end{array}$ & 1 & $22.601,754$ & 0,0001 & 19,23 & 26,4054 & 0,0001 & 20,98 \\
\hline $\begin{array}{l}\text { Resíduo } \\
\text { Error }\end{array}$ & 5014 & 7,353 & & & 0,0079 & & \\
\hline$\overline{\mathrm{CV}}(\%)$ & 18,02 & & & 17,66 & & & \\
\hline
\end{tabular}


1020 Rev. bras. zootec.

\section{SCHAEFFER e HENDERSON (1972), SMITH e} LEGATES (1962) e THOMPSON et al. (1982) encontraram relações quadráticas e TEIXEIRA et al. (1995) obtiveram relações cúbicas. No caso dos resultados obtidos por esses autores, foram utilizados períodos de serviço mais longos (até acima de 229 dias) em relação aos considerados neste trabalho (período de serviço máximo de 229 dias). As relações entre LDIA e PSER também foram descritas por equações lineares decrescentes (Figura 2), sendo semelhantes às relações entre GDIA e PSER. Pela análise das Figuras 1 e 2, pode-se observar que, com o aumento do período de serviço, ocorreu redução

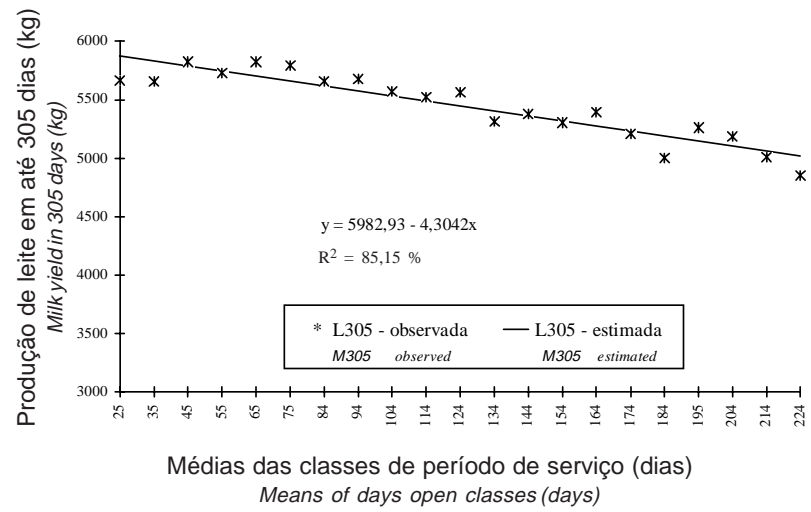

Figura 1 - Produção de leite estimada em até 305 dias, de acordo com as médias das classes de período de serviço.

Figure 1 - Estimated milk yield in 305 days according to the means of the days open classes.

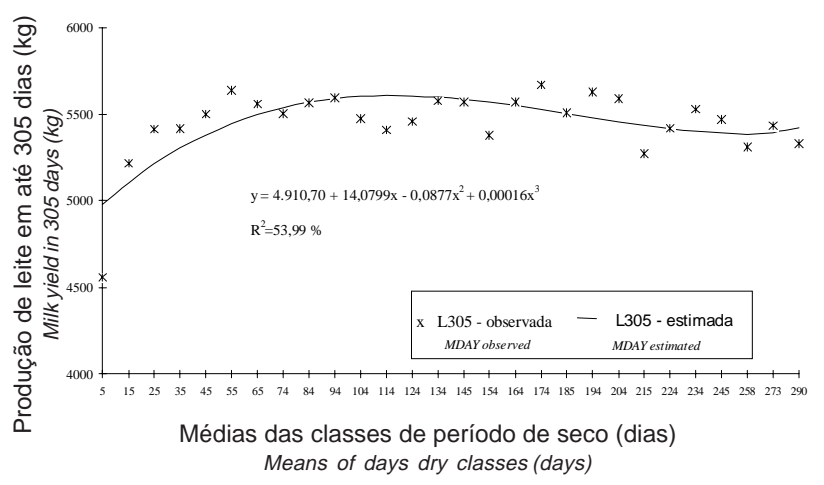

Figura 3 - Produção de leite estimada em até 305 dias, de acordo com as médias das classes de período seco anterior.

Figure 3 - Estimated milk yield in 305 days according to the means of the previous days dry classes. mais acentuada em LDIA que em L305, o que é esperado, tendo em vista que maior PSER proporciona aumento no intervalo de partos, reduzindo LDIA, o mesmo ocorrendo com GDIA.

As relações entre produção de leite em até 305 dias e produção de leite por dia de intervalo de parto com o período seco anterior em classes apresentaram tendência cúbica (Figuras 3 e 4), o mesmo ocorrendo para as relações entre G305 e GDIA com PSA, semelhante aos resultados obtidos por TEIXEIRA et al. (1995) no caso da relação entre L305 e período seco anterior.

A justificativa para este relacionamento é comple-

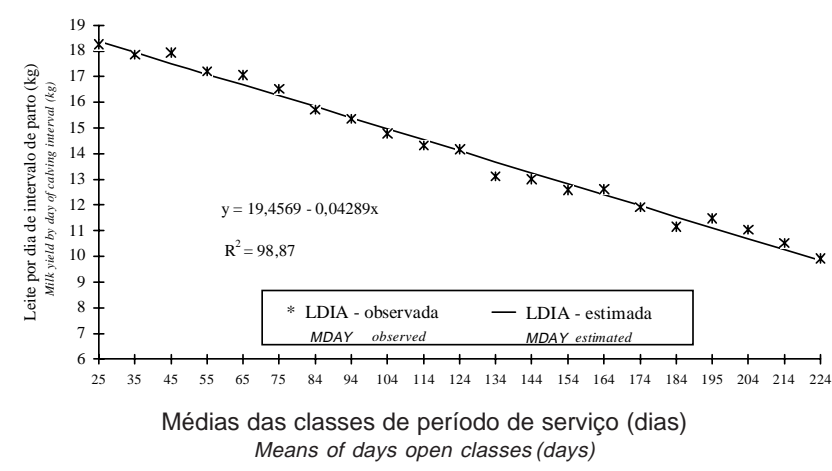

Figura 2 - Produção de leite estimada por dia de intervalo de parto, de acordo com as médias das classes de período de serviço.

Figure 2 - Estimated milk yield by day of calving interval according to the means of the days open classes.

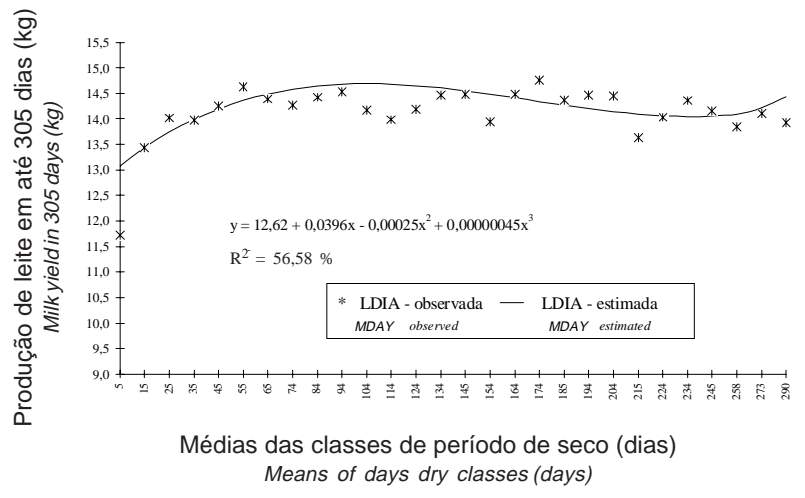

Figura 4 - Produção de leite estimada por dia de intervalo de parto, de acordo com as médias das classes de período seco anterior.

Figure 4 - Estimated milk yield by day of calving interval according to the means of the previous days dry classes. 
OLIVEIRA et al.

xa, devido à alta variabilidade das produções em função do PSA, a qual pode ser observada pelos baixos valores de coeficiente de determinação das curvas cúbicas, que se ajustaram aos dados (Figuras 3 e 4). Tendo em vista que aumento no PSA proporciona maior descanso fisiológico do animal, esperar-se-ia aumento quadrático na produção de leite, semelhante ao obtido por TEIXEIRA et al. (1994); contudo, foi obtido apenas ligeiro aumento até os 60-70 dias de PSA.É possível que o fato de se ter considerado PSA de até 300 dias tenha levado à obtenção da relação cúbica.

A Tabela 4 apresenta fatores de ajuste para a L305 e LDIA com base no período de serviço. Os resultados evidenciam maior influência sobre LDIA em relação L305, sendo importante considerar que os registros referentes à produção por dia de intervalo, quando forem utilizados na seleção de touros ou vacas, deverão ter seus valores ajustados.

Tabela 4 - Fator de ajuste multiplicativo, classe de período de serviço com respectivos períodos médios de serviço e média de L305 e LDIA

Table 4 - Multiplicative adjust factor, days open class with respective days open means and average of M305 and MDAY

\begin{tabular}{|c|c|c|c|c|c|}
\hline \multicolumn{2}{|c|}{$\begin{array}{c}\text { Período de serviço } \\
\text { Days open }\end{array}$} & \multirow[t]{3}{*}{$\begin{array}{c}\mathrm{L} 305(\mathrm{~kg}) \\
\text { M305 }\end{array}$} & \multirow[t]{3}{*}{$\begin{array}{c}\text { Fator de ajuste } \\
\text { Adjustfactor }\end{array}$} & \multirow[t]{3}{*}{$\begin{array}{c}\text { LDIA }(\mathrm{kg}) \\
M D A Y\end{array}$} & \multirow[t]{3}{*}{$\begin{array}{r}\text { Fator de ajuste } \\
\text { Adjustfactor }\end{array}$} \\
\hline Classe (dias) & Média & & & & \\
\hline Class (days) & Mean & & & & \\
\hline $20-29$ & 25 & 5666,70 & 1,0697 & 18,26 & 1,2620 \\
\hline $30-39$ & 35 & 5654,72 & 1,0619 & 17,86 & 1,2326 \\
\hline $40-49$ & 45 & 5824,73 & 1,0541 & 17,94 & 1,2032 \\
\hline $50-59$ & 55 & 5731,54 & 1,0462 & 17,21 & 1,1737 \\
\hline $60-69$ & 65 & 5826,78 & 1,0384 & 17,07 & 1,1443 \\
\hline $70-79$ & 75 & 5794,70 & 1,0306 & 16,53 & 1,1148 \\
\hline $80-89$ & 84 & 5657,23 & 1,0235 & 15,72 & 1,0883 \\
\hline $90-99$ & 94 & 5676,42 & 1,0157 & 15,36 & 1,0589 \\
\hline $100-109$ & 104 & 5572,54 & 1,0078 & 14,79 & 1,0294 \\
\hline $110-119$ & 114 & 5521,77 & 1,0000 & 14,33 & 1,0000 \\
\hline $120-129$ & 124 & 5564,65 & 0,9922 & 14,16 & 0,9706 \\
\hline $130-139$ & 134 & 5311,69 & 0,9843 & 13,12 & 0,9411 \\
\hline $140-149$ & 144 & 5375,72 & 0,9765 & 13,02 & 0,9117 \\
\hline $150-159$ & 154 & 5305,99 & 0,9687 & 12,58 & 0,8822 \\
\hline $160-169$ & 164 & 5396,27 & 0,9608 & 12,63 & 0,8528 \\
\hline $170-179$ & 174 & 5209,11 & 0,9530 & 11,91 & 0,8233 \\
\hline 180-189 & 184 & 5004,25 & 0,9451 & 11,15 & 0,7939 \\
\hline 190-199 & 195 & 5259,31 & 0,9365 & 11,49 & 0,7615 \\
\hline $200-209$ & 204 & 5187,29 & 0,9295 & 11,04 & 0,7350 \\
\hline $210-219$ & 214 & 5010,24 & 0,9216 & 10,52 & 0,7056 \\
\hline $220-229$ & 224 & 4851,47 & 0,9138 & 9,93 & 0,6761 \\
\hline
\end{tabular}

\section{Conclusões}

O ajustamento das L305, G305, LDIA e GDIA para período de serviço tende a melhorar as avaliações genéticas para estas produções. Adicionalmente, é de grande interesse a realização de mais pesquisas para esclarecer se a contribuição destas características é genética ou ambiental.

\section{Referências Bibliográficas}

KHATTAB, A.S., ASHMAWY, A.A. 1988. Relationships of days dry and days dry with milk production in Friesian cattle in Egypt. J. Anim. Breeding Genetics, 105(4):300-305.

LOUCA, A., LEGATES, J.E. 1968. Production losses in dairy cattle due to days open. J. Dairy Sci., 51(4):573-583.

OLIVEIRA FILHO, E.B., LOBO, R.B., DUARTE, F.A. 1985. Eficiência reprodutiva de vacas Gir exploradas para leite. Rev. Bras. Reprod. Anim., 9(1):21-33. 
1022 Rev. bras. zootec.

SCHAEFFER, L.R., HENDERSON, C.P. 1972. Effects of days dry and days open on Holstein milk production. J. Dairy Sci., 55(1):107-112.

SMITH, J.W., LEGATES, J.E. 1962. Relation of days open and days dry to lactation milk and fat yields. J. Dairy Sci., 45:1192-1197.

TEIXEIRA, N.M., RIBAS, N.P., VALENTE, J. et al. Influência dos períodos seco e de serviço sobre a produção de leite em 305 dias no gado Holandês. In: REUNIÃO ANUAL DA SOCIEDADE BRASILEIRA DE ZOOTECNIA, 31, 1994, Maringá. Anais... Maringá: SBZ/UEM, 1994, p.222.

TEIXEIRA, N.M., VALENTE, J. Efeito dos períodos seco anterior e de serviço corrente sobre a produção de leite anualizada no gado Holandês. In: REUNIÃO ANUAL DA SOCIEDADE BRASILEIRA DE ZOOTECNIA, 31, 1994, Maringá. Anais... Maringá: SBZ/UEM, 1994, p.223.

TEIXEIRA, N.M., VALENTE, J., FREITAS, A.F. et al. Influências dos períodos de serviço e seco sobre a produção de leite em 305 dias na raça Holandesa. In: REUNIÃO ANUAL DA SOCIEDADE BRASILEIRA DE ZOOTECNIA, 32, 1995,
Brasília. Anais... Brasília: SBZ, p.706-708, 1995.

THOMPSON, J.R., FREEMAN, A.E., BERGER, P.J. 1982. Days open adjusted, annualized, and fat-corrected yields as alternatives to mature-equivalent records. J. Dairy Sci., 65(8):1562-1577.

VALENTE, J., TEIXEIRA, N.M., VERNEQUE, R.S. da. et al. Efeitos dos períodos de serviço anterior, período seco anterior e período de serviço corrente sobre a produção de leite. In: REUNIÃO ANUAL DA SOCIEDADE BRASILEIRA DEZOOTECNIA, 32, 1995, Brasília. Anais... Brasília: SBZ, p.686-688, 1995.

Recebido em: 15/10/96

Aceito em: 22/04/99 